\title{
Enumerating lepidopteran species associated with maize as a first step in risk assessment in the USA
}

\author{
John E. LOSEY ${ }^{1, \star}$, Ruth A. HUFBAUER ${ }^{2}$, Robert G. HARTZLER ${ }^{3}$ \\ ${ }^{1}$ Department of Entomology, Cornell University, Ithaca, NY 14853, USA \\ ${ }^{2}$ Department of Bioagricultural Sciences and Pest Management, Colorado State University, Fort Collins, CO 80523, USA \\ ${ }^{3}$ Department of Agronomy, Iowa State University, IA 50011, USA
}

\begin{abstract}
Pest management can have substantial impacts on non-target species both within and outside the units being managed. Assessment of these impacts is hampered by the lack of even the most basic checklist of the species present in most systems. The maize agroecosytem is of particular interest because of the large area covered and the intensity of widely varying forms of pest management. In this study a database of lepidopteran species that occur within the maize agroecosystem in the United States was compiled. The process was initiated by developing a list of plants present in maize using published sources and the first-hand knowledge of "weed" experts. This list of plant species associated with maize was then cross-listed with lepidopteran host feeding records using published sources. Finally, phenological profiles and conservation rankings were added. Although our list is not exhaustive, we found 132 plant species in 33 families associated with maize, and 229 lepidopteran species in 21 families that feed on these plants. The database of plants and lepidopteran species can be a starting point for assessment of risk to non-target Lepidoptera in maize from chemical control, biological control, and the use of transgenic Bt maize. The lepidopteran species associated with maize were found to be significantly less imperiled, as measured by their conservation rankings, than lepidopteran species as a whole in all habitats. This finding suggests that rare or endangered lepidopteran species are unlikely to be impacted by pest management in maize. Based on the likely lack of impact of pest management in maize on individual species, future studies should focus on potential impacts on the ecological services that lepidopteran species provide.
\end{abstract}

Keywords: Bt maize / transgenic / GMO / non-target species / risk assessment

\section{INTRODUCTION}

Pest management and conservation are the two major endeavors involved with the management of insect populations. Although they are often considered separate or even in conflict with each other, these two disciplines are inextricably linked and interdependent. The goal of insect pest management is to maintain insect pest species below threshold densities. Pest management tactics interfere with the ability of insects to survive, reproduce or exploit resources, and the impacts of these tactics are very rarely confined to the target pest species. These relatively broad effects clearly impinge on a substantial proportion of insect habitats since, $70 \%$ of the land on earth is utilized for agriculture and forestry and presumably receives some level of pest management (Western, 1989). Two main goals of insect conservation are the preservation of rare insect species and of the ecological services that insect populations provide. Clearly, pest management practices can have important impacts on the conservation of insects and conversely, most managed systems could not function without the ecological services such as pollination, the biological control of pests, and decomposition of plant and animal tissue that

\footnotetext{
* Corresponding author:

Tel.: (607) 255-7376; fax: (607) 255-0939; e-mail: jel27@ cornell.edu
} 
insects provide. The first step towards a comprehensive insect management program that would provide adequate pest suppression, maintenance of ecological services, and minimal impact on rare species is a detailed assessment of which insect species are likely to exist in the managed system. Unfortunately, this baseline accounting of insect species is lacking for almost every managed system.

Here we describe a portion of the insect community associated with maize in the United States. The maize system is of particular interest because it outranks all other cultivated crops in the USA in terms of overall value, land area covered, and total pesticides used (U.S. Grains Council, 2002). Over 240 million metric tons of maize were produced in the USA in 2001, which was just over $41 \%$ of worldwide maize production (FAS-USDA, 2002). In the USA, maize is valued at \$ 19 billion/year, it is grown on 32 million hectares, and over 100 million kilograms of pesticides are used annually (Economic Research ServiceUSDA, 2002; Padgitt et al., 2001). Pesticide usage consists primarily of insecticides that can directly impact non-target insects and herbicides that can impact insects indirectly by removing host plants. Considering the size of the maize system and the intensity of pest management applied to it, the potential for substantial impacts on nontarget insect species and services would appear to be high. Insects provide important ecological services in the maize system including contribution to the biological control of key pest insects and weeds.

The large area covered by maize production and the broad range of ecoregions encompassed would make an accounting of all insect species impractical for the scope of a single study. In this study we focus on species in the order Lepidoptera. The Lepidoptera were chosen because they include some of the major maize pests (e.g., Ostrinia nubilalis, and Heliocoverpa zea) and this taxon also contains a substantial number of rare or endangered species. In addition, one of the few pest management tactics that is specific to an insect order, the use of transgenic maize expressing a gene encoding a Bacillus thuringiensis endotoxin (hereafter referred to as $B t$ maize), is specific to Lepidoptera. Since Lepidoptera are generally not predaceous or parasitic, they do not contribute to the suppression of pest insects. Although there are few data on the ecological roles of most Lepidoptera in maize, it has been documented across several systems that many lepidopteran species contribute to the biological control of important weed species (Julien and Griffiths, 1998), and they provide alternate prey for the natural enemies of important pests (Biddinger et al., 1994; Pavuk and Stinner, 1991). An accounting of Lepidoptera in maize will greatly facilitate the design of management plans that provide adequate pest suppression while maintaining ecological services and minimizing effects on rare species. The objective of this study is to provide a baseline list of the lepidopteran species that are likely to occur in and around maize in the continental USA.

\section{RESULTS}

The survey of plants associated with maize identified 132 species in 33 families. The plant families accounting for the most species were Poaceae with 28 and Asteraceae with 20 (Appendix 1). Several plant families were represented by a single species. More than half the species $(51.5 \%)$ were ranked as rare in maize, while $35.6 \%$ were ranked as common and $12.9 \%$ were ranked as abundant.

The survey of lepidopteran species that feed on plants associated with maize identified 229 species in 21 families (Appendix 2). The lepidoteran families accounting for the most species were Noctuidae with 84 and Hesperidae with 31. Several lepidopteran families were represented by a single species. Approximately one quarter of the lepidopteran species was identified as feeding on more than one plant species.

The distributions of global and national conservation status ranks for lepidopteran species identified as associated with maize were significantly different than the distributions of ranks for all lepidopteran species ranked in the NatureServe database $(P<0.0001$; Figs. 1 and 2). Higher proportions of lepidopteran species associated with maize were assigned higher ranks (denoting a more secure status) compared to the proportions of all ranked lepidopteran species. Specifically, $87.4 \%$ of maize species were assigned a rank of G5 (globally secure) and $83.7 \%$ were assigned a rank of N5 (nationally secure), compared with $55.4 \%$ and $27.9 \%$, respectively, for all the lepidopteran species ranked in the NatureServe database.

Only one maize-associated species, the mottled duskywing, Erynnis martialis (Lepidoptera: Hesperiidae), was assigned a rank below G4, denoting the species is at risk globally. It is important to note that although the karner blue butterfly, Lycaeides melissa samuelis (Lepidoptera: Lycaenidae), is ranked at G5, it is arguably more endangered than the mottled duskywing. Both species occur on host plants that are associated with maize but the largest stands of karner blue's primary host, wild lupine, grow in pine/oak savannahs that are rapidly disappearing due to fire suppression and development. Based on the rapid decline in its habitat the karner blue has been placed on the United States endangered species list. Both the karner blue and the monarch butterfly, 


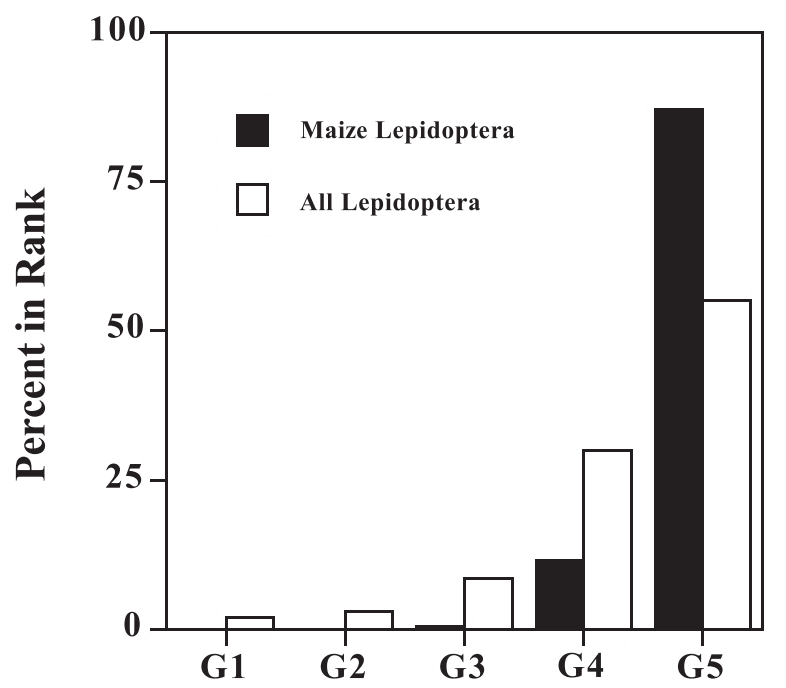

Global Ranks

Figure 1. Frequency distribution for global conservation ranks of lepidopteran species. Black bars represent ranks of species associated with maize, and white bars represent species from all habitats combined.

Danaus plexippus (Lepidoptera: Nymphalidae), were assigned national ranks of N2 (nationally imperiled). The mottled duskywing and the southern scalloped sooty wing, Staphylus mazans (Lepidoptera: Hesperiidae), were assigned ranks of N3 (nationally vulnerable). As illustrated by these examples, national ranks are often lower than global ranks because they represent a subset of all the populations of a species. Thus a species could be imperiled in several individual countries yet globally secure. All other ranked species associated with maize were assigned ranks of N4 or N5 that denote apparent or demonstrable security nationally.

\section{DISCUSSION}

The results of this survey show the unexpected complexity and diversity associated with the maize agroecosystem. Specifically they indicate clearly that there is a substantial number of lepidopteran species that feed on plants in and around fields of maize. This list of species can provide the basis for assessment of risk from a pest management tactic. Using this data set, species can be selected for more in-depth risk assessment based on their phenology and their security status. For example, univoltine species that go through their development in June could be given low priority for tactics applied in July and imperiled species

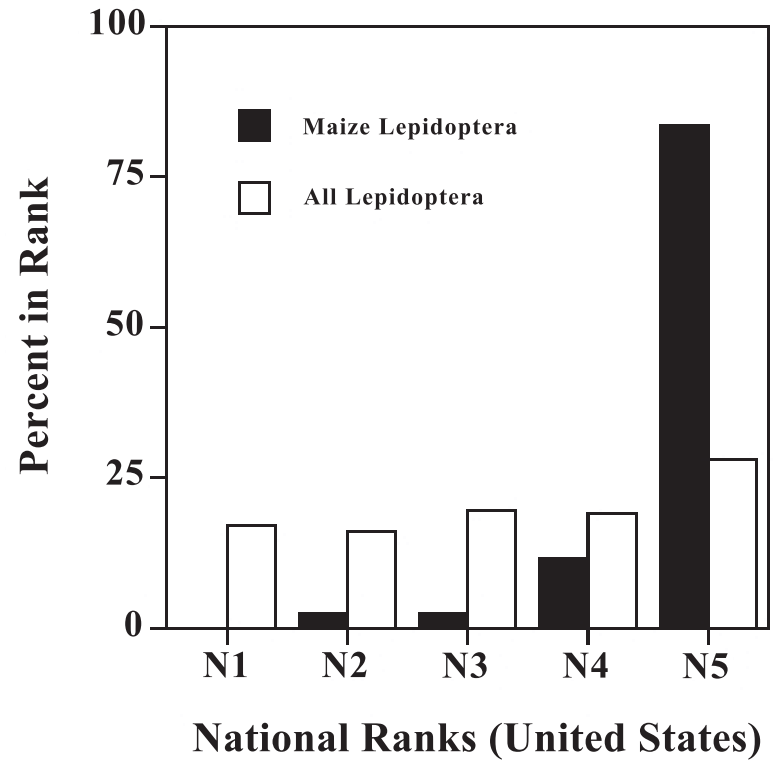

Figure 2. Frequency distribution for United States national conservation ranks of lepidopteran species. Black bars represent ranks of species associated with maize, and white bars represent species from all habitats combined.

with low security ranks could be given higher priority than those with higher security status ranks.

When considering either the list of species or their relative security potential, sources of bias should be considered. Lepidopteran checklists are probably likely to have a bias towards more common species, while the data on species security is more complete for less secure species that are usually rare. Groups such as the microlepidoptera that have not been well studied are almost certainly underrepresented in most sources. While each individual source may miss several rare species, by consulting a large number of sources we are confident that we have assembled sufficient data to firmly ground our conclusions.

Once a group of species is selected, risk assessment could proceed by gathering data on: (1) the relative importance of maize as a habitat for the host plant; (2) the relative importance of that plant for the herbivore species; and (3) the susceptibility of the species to impact from the management tactic being assessed. Further information may be available on the relative importance of maize as a habitat for the host plant. Because most non-crop plants in and around maize fields are considered weeds, the make-up of these plant communities is fairly well known. Unfortunately, what is not known for most of the plants associated with maize is the proportion of their total 
distribution that falls within a distance that is likely to be affected by a given pest management tactic. An additional complicating factor is that the adoption of reduced tillage systems has increased the diversity of plants occurring in agricultural fields (Cardina et al., 1991).

It is possible to gauge the relative importance of a given set of host plants for a lepidopteran species by more in-depth scrutiny of the species life history. Specialist herbivores feeding on plants that grow exclusively near maize fields are much more likely to be affected by pest management in maize than herbivores that feed on plants growing in several habitats, in addition to maize. This type of niche analysis for a large number of species would not be trivial, so it is probably best reserved for those species that are identified as high priority in the initial screen.

By combining the relative importance of maize as a habitat for the host plant and the relative utilization of that host plant by the herbivore species, it is possible to estimate the importance of maize fields as a habitat for the lepidopteran species being examined. The data gathered will be equally applicable to any pest management tactic. Conversely, data on susceptibility will be almost completely specific to the tactic being assessed. There is a large amount of published data available on the susceptibility of many lepidopteran species to chemical applications and smaller amounts of data available on the $B t$ toxin that is incorporated into transgenic maize (Johnson et al., 1995; Peacock et al., 1998), and the egg parasitoid Trichogramma that is used as an augmentative biological control agent (Hoffmann et al., 1995; Orr et al., 2000). Unfortunately, an assessment of the susceptibility of all the lepidopteran species associated with maize is not available for any of the tactics. In general, most lepidopteran species are physiologically susceptible to the insecticides used to control lepidopteran pests in maize. However, susceptibility to chemical insecticides is dependent on relative size and feeding niche. Small larvae feeding on exposed leaves will be more susceptible than large larvae feeding in a protected area like the roots or stalk of a plant.

In contrast to insecticides, physiological susceptibility to the $B t$ toxin varies widely within lepidopteran families and even within genera (Johnson et al., 1995; Peacock et al., 1998). This extreme variability essentially makes it impossible to establish a phylogenetic pattern that could guide the risk assessment process. Thus, a full assessment of any lepidopteran species for which no data on susceptibility to Bt exists may require standardized toxicity testing.

Among the three main tactics the fewest data exist for susceptibility to Trichogramma wasps. The challenge in assessing risk for Trichogramma is that laboratory measures of physiological susceptibility (e.g. tests of oviposition in the eggs of a particular lepidopteran species) do not necessarily lead to accurate predictions of the level of non-target risk in the field. A current study, utilizing the database presented here, is assessing both the physiological and ecological susceptibility of a large group of lepidopteran species associated with maize to Trichogramma ostriniae (Wright et al., unpublished).

In addition to providing a list and biological information for more in-depth risk assessment, our review also uncovered an emergent property which applies generally to the complex of lepidopteran species that are associated with maize: only a very small proportion of the complex of lepidopteran species associated with maize are rare or endangered. In fact, this complex of species appears to have a lower proportion of rare or endangered species than the Lepidoptera as a whole. This is probably due to the large area covered by maize in a wide range of ecoregions and the fact that most of the plants associated with maize (e.g. weeds) are also adapted to thrive in other disturbed areas including other crops. In general, based on the prevalence of the maize habitat, any Lepidopteran that feeds on a plant associated with maize is probably not in immediate peril. The implication of this emergent property is that pest management tactics applied to maize are unlikely to affect an endangered or threatened lepidopteran species associated with non-maize plants that grow in and around maize fields.

The predicted lack of effects on endangered lepidopteran species does not mean that pest management tactics will not have negative impacts on the lepidopteran complex in maize as a whole. Many common lepidopteran species provide invaluable services such as the biological control of weeds (Julien and Griffiths, 1998), pollination (Buchmann and Nabhan, 1996), and alternate hosts for parasitoids of insect pests (Biddinger et al., 1994; Pavuk and Stinner, 1991). If pest management tactics depress lepidopteran densities, they may interfere with the provision of these services. If maize fields are serving as sources of the species providing these services in other systems, then even local depression of lepidopteran densities may cause disruption of the services on a regional basis. Thus, further assessment of the risk to the lepidopteran species associated with maize should focus on functional guilds as well as individual species.

\section{MATERIALS AND METHODS}

The first step in any risk assessment is developing a list of species that might be affected by the pest management 
tactics employed. Once a complete list is generated the relative level of risk can be estimated for each individual species. Our list is restricted to the top 17 maize producing states in the United States which are (in order of hectares planted to maize) IA, IL, NE, MN, IN, SD, WI, OH, KS, MO, MI, TX, PA, KY, CO, and NY. These 17 states account for over $90 \%$ of the maize planted in the United States (National Agricultural Statistics Service USDA, 2000).

Since Lepidoptera are almost universally herbivorous, our first step in generating a list of lepidopteran species associated with maize was to determine which plant species were likely to be associated with this crop. The list of plant species in maize was generated through a combination of published data and personal knowledge (RGH) of plants in the maize system. The list is not intended to be exhaustive, but it does include most of the common and abundant plants, and illustrates the wide variety of plants associated with maize production. Each plant species was ranked as abundant, common, or rare by RGH.

The next step in our sequential approach was to determine which lepidopteran species feed on plants within the maize system. By cross-listing the plant species with the lepidopteran species that feed on these plants, an initial list of non-target herbivores was generated. All records of lepidopteran feeding on individual host plant species are based on published sources.

Once it had been determined which lepidopteran species feed on plant species in the maize system, the next step was to determine which of those herbivore species are feeding in the larval stage during the period when pest management tactics are likely to affect them. Since most pest management that would affect lepidopteran species in USA maize fields is undertaken in the summer months, we restricted our phenological profile to larval presence/absence in June, July and August. This phenological overlap with pest management tactics can serve as one measure of the potential severity of risk to individual species. The "effective" period is well known for most pest management tactics. To be affected by a given tactic, larvae must be present during or immediately after this period. Although exact phenological data are not available for many of the lepidopteran species identified, it is often possible to determine which of the summer months the larvae of most species are known to be active.
The final step in our protocol was the inclusion of a relative imperilment or security status ranking for all species currently ranked by NatureServe (2001). These ranks are calculated through consultation of both published sources and a network of taxon specific expertise (NatureServe, 2001). The conservation rank of a species known or assumed to exist within a jurisdiction is designated by a whole number from 1 to 5 , preceded by $\mathrm{G}$ (Global) or $\mathrm{N}$ (National). The ranks have the following meaning: $1=$ critically imperiled; $2=$ imperiled; $3=$ vulnerable to extirpation or extinction; $4=$ apparently secure; and 5 = demonstrably widespread, abundant, and secure. G1, for example, indicates critical imperilment on a range-wide basis while $\mathrm{N} 1$ indicates critical imperilment within the United States. These rankings provide a measure of which potentially affected species should be of particular concern.

Global and national ranks for Lepidoptera identified in our study as being associated with maize were compared with ranks of all the lepidopteran species (across all habitats) from the NatureServe database (NatureServe, 2001). Species with a rank range were assigned the lower rank. For example, a species ranked as G3G4 were assigned a rank of G3 for this analysis. Comparisons were analyzed with the two-tailed Fisher's exact chisquare test, PROC FREQ (SAS Institute, 1996).

\section{ACKNOWLEDGEMENTS}

The authors wish to thank Catherine Day, Megan Edlund, Melissa Jensen, Stephanie Johnson, Luisa Perez, and Diane Tran for their assistance with gathering and collating data for the database. We also wish to thank Robert Dirig for his helpful early comments on lepidopteran species and their host plants and Richard Hoebeke for his help with deciphering nomenclature. The manuscript was greatly improved by editorial input from Leslie Allee. Data on conservation ranks was provided with permission and assistance from NatureServe: Copyright ${ }^{\circledR} 2001$ NatureServe, 1101 Wilson Boulevard, 15th Floor, Arlington, Virginia 22209, USA. All Rights Reserved.

Received October 8, 2002; accepted July 15, 2003. 
Appendix 1.

Plants associated with maize that are potential hosts of lepidopteran species.

\begin{tabular}{|c|c|c|c|c|c|}
\hline $\begin{array}{l}\text { Plant } \\
\text { Number }\end{array}$ & Plant Common Name & Plant Scientific Name & Plant Family & Abundance & $\begin{array}{c}\text { Weed } \\
\text { Reference }\end{array}$ \\
\hline 1 & Tumble piweed & Amaranthus albus & Amaranthaceae & Common & 1 \\
\hline 2 & Sandhills amaranth & Amaranthus arenicola & Amaranthaceae & Rare & 1 \\
\hline 3 & Prostrate pigweed & Amaranthus graecizans & Amaranthaceae & Rare & - \\
\hline 4 & Palmer amaranth & Amaranthus palmeri & Amaranthaceae & Common & 1 \\
\hline 5 & Red root pigweed & Amaranthus retroflexus & Amaranthaceae & Abundant & 2 \\
\hline 6 & Common waterhemp & Amaranthus rudis & Amaranthaceae & Abundant & - \\
\hline 7 & Spiny amaranth & Amaranthus spinosus & Amaranthaceae & Rare & - \\
\hline 8 & Wild carrot & Daucus carota & Apiaceae & Rare & - \\
\hline 9 & Hemp dogbane & Apocynum cannabinum & Apocynaceae & Common & 2 \\
\hline 10 & Common milkweed & Asclepias syriaca & Asclepiadacea & Common & 2 \\
\hline 11 & Honeyvine milkweed & Cynanchum laeve & Asclepiadacea & Common & - \\
\hline 12 & Black swallow-wort & Vincetoxicum nigrum & Asclepiadacea & Common & - \\
\hline 13 & Common ragweed & Ambrosia artemisiifolia & Asteraceae & Abundant & - \\
\hline 14 & Lanceleaf ragweed & Ambrosia bidentata & Asteraceae & Rare & - \\
\hline 15 & Western ragweed & Ambrosia psilostachya & Asteraceae & Rare & - \\
\hline 16 & Giant ragweed & Ambrosia trifida & Asteraceae & Abundant & - \\
\hline 17 & Common burdock & Arctium minus & Asteraceae & Rare & - \\
\hline 18 & Musk thistle & Carduus nutans & Asteraceae & Rare & - \\
\hline 19 & Tall thistle & Cirsium altissimum & Asteraceae & Rare & - \\
\hline 20 & Canada thistle & Cirsium arvense & Asteraceae & Common & 2 \\
\hline 21 & Bull thistle & Cirsium vulgare & Asteraceae & Rare & - \\
\hline 22 & Horseweed & Conyza canadensis & Asteraceae & Common & - \\
\hline 23 & Rough fleabane & Erigeron strigosus & Asteraceae & Rare & - \\
\hline 24 & Small flower galinsoga & a Galinsoga parviflora & Asteraceae & Rare & - \\
\hline 25 & Common sunflower & Helilanthus annuиs & Asteraceae & Abundant & 2 \\
\hline 26 & Sawtooth sunflower & Helilanthus grosseserratus & Asteraceae & Rare & - \\
\hline 27 & Jerusalem artichoke & Helilanthus tuberosus & Asteraceae & Common & - \\
\hline 28 & Marsh elder & Iva xanthifolia & Asteraceae & Rare & 3 \\
\hline 29 & Gray goldenrod & Solidago nemoralis & Asteraceae & Rare & - \\
\hline 30 & Perennial sowthistle & Sonchus arvensis & Asteraceae & Rare & - \\
\hline 31 & Dandelion & Taraxacum officinale & Asteraceae & Common & - \\
\hline 32 & Common cocklebur & Xanthium strumarium & Asteraceae & Abundant & 2 \\
\hline 33 & Trumpet creeper & Campsis radicans & Bignoniaceae & Rare & 2 \\
\hline 34 & Yellow rocket & Barbarea vulgaris & Brassicaceae & Common & - \\
\hline 35 & Indian mustard & Brassica juncea & Brassicaceae & Common & - \\
\hline 36 & Wild mustard & Brassica kaber & Brassicaceae & Common & - \\
\hline 37 & Black mustard & Brassica nigra & Brassicaceae & Rare & - \\
\hline 38 & Shepherd's purse & Capsella bursa-pastoris & Brassicaceae & Common & - \\
\hline 39 & Blue mustard & Chorispora tenella & Brassicaceae & Common & - \\
\hline 40 & Field pepperweed & Lepidium campestre & Brassicaceae & Common & - \\
\hline 41 & Tallhedge mustard & Sisymbrium loeselii & Brassicaceae & Common & - \\
\hline 42 & Field pennycress & Thlaspi arvense & Brassicaceae & Common & - \\
\hline 43 & Marijuana & Cannabis sativa & Cannabaceae & Common & - \\
\hline 44 & Corn cockle & Agrostemma githago & Caryophyllaceae & Rare & - \\
\hline 45 & Mouse-ear chickweed & Cerastium vulgatum & Caryophyllaceae & Rare & - \\
\hline 46 & Bouncingbet & Saponaria officinalis & Caryophyllaceae & Rare & - \\
\hline 47 & White cockle & Silene alba & Caryophyllaceae & Rare & - \\
\hline 48 & Common chickweed & Stellaria media & Caryophyllaceae & Common & - \\
\hline 49 & Spreading orach & Atriplex subspicata & Chenopodiaceae & Rare & - \\
\hline 50 & Common lambsquarter & Chenopodium album & Chenopodiaceae & Abundant & 2,4 \\
\hline 51 & Mapleleaf goosefoot & Chenopodium simplex & Chenopodiaceae & Rare & - \\
\hline 52 & Kochia & Kochia scoparia & Chenopodiaceae & Common & - \\
\hline 53 & Rusian thistle & Salsola iberi ca & Chenopodiaceae & Common & 3 \\
\hline 54 & Hedge bindweed & Calystegia sepium & Convolvulaceae & Common & - \\
\hline 55 & Field bindweed & Convolvulus arvensis & Convolvulaceae & Common & - \\
\hline 56 & Bindweeds & Convolvulus spp. & Convolvulaceae & Common & - \\
\hline 57 & Ivyleaf morning glory & Ipomoea hederacea & Convolvulaceae & Common & - \\
\hline 58 & Purple morning glory & Iротоеа ригригеа & Convolvulaceae & Common & 1 \\
\hline
\end{tabular}


Lepidopteran species associated with maize

\begin{tabular}{|c|c|c|c|c|c|}
\hline $\begin{array}{l}\text { Plant } \\
\text { Number }\end{array}$ & Plant Common Name & Plant Scientific Name & Plant Family & Abundance & $\begin{array}{c}\text { Weed } \\
\text { Reference }\end{array}$ \\
\hline 59 & Buffalo gourd & Cucurbita foetidissima & Cucurbitaceae & Rare & - \\
\hline 60 & Wild cucumber & Echinocystis lobata & Cucurbitaceae & Common & - \\
\hline 61 & Bur cucumber & Sicyos angulatus & Cucurbitaceae & Common & - \\
\hline 62 & Yellow nutsedge & Cyperus rotundus & Cyperaceae & Abundant & 1 \\
\hline 63 & Field horsetail & Equisetum arvense & Equisetaceae & Rare & - \\
\hline 64 & Virginia copperleaf & Acalypha virginica & Euphorbiaceae & Rare & - \\
\hline 65 & Toothed spurge & Euphorbia dentata & Euphorbiaceae & Common & 3 \\
\hline 66 & Spotted spurge & Euphorbia maculata & Euphorbiaceae & Rare & - \\
\hline 67 & Alfalfa & Medicago sativa & Fabaceae & Rare & \\
\hline 68 & White clover & Trifolium repens & Fabaceae & Rare & \\
\hline 69 & Carolina geranium & Geranium carolinianum & Geraniaceae & Rare & - \\
\hline 70 & Wild onion & Allium canadense & Liliaceae & Rare & - \\
\hline 71 & Wild garlic & Allium vineale & Liliaceae & Rare & - \\
\hline 72 & Velvetleaf & Abutilon theophrasti & Malvaceae & Abundant & 2 \\
\hline 73 & Venice mallow & Hibiscus trionum & Malvaceae & Common & 3 \\
\hline 74 & Common mallow & Malva neglecta & Malvaceae & Rare & 3 \\
\hline 75 & Prickly sida & Sida spinosa & Malvaceae & Common & - \\
\hline 76 & Carpetweed & Mollugo verticillata & Molluniginaceae & Rare & - \\
\hline 77 & Wild four-o'clock & Mirabilis nyctaginea & Nyctaginaceae & Rare & - \\
\hline 78 & Evening primrose & Oenothera biennis & Onagraceae & Rare & - \\
\hline 79 & Yellow woodsorrel & Oxalis stricta & Oxalidaceae & Rare & - \\
\hline 80 & Common pokeweed & Phytolacca americana & Phytolaccaceae & Rare & - \\
\hline 81 & Buckhorn plantain & Plantago lanceolata & Plantaginaceae & Rare & - \\
\hline 82 & Quackgrass & Agropyron repens & Poaceae & Abundant & - \\
\hline 83 & Wild oat & Avena fatua & Poaceae & Rare & 3 \\
\hline 84 & Signalgrass & Brachiaria platyphylla & Poaceae & Rare & 1 \\
\hline 85 & Japanese brome & Bromus japonicus & Poaceae & Rare & - \\
\hline 86 & Cheatgrass & Bromus secalinus & Poaceae & Rare & - \\
\hline 87 & Downy brome & Bromus tectorum & Poaceae & Rare & - \\
\hline 88 & Longspine sandbur & Cenchrus longispinus & Poaceae & Common & 3 \\
\hline 89 & Bermudagrass & Cynodon dactylon & Poaceae & Rare & 1 \\
\hline 90 & Orchardgrass & Dactylis glomerata & Poaceae & Rare & - \\
\hline 91 & Smooth crabgrass & Digitaria ischaemum & Poaceae & Common & 1 \\
\hline 92 & Large crabgrass & Digitaria sanguinalis & Poaceae & Common & 1 \\
\hline 93 & Barnyardgrass & Echinochloa crus-galli & Poaceae & Common & 3 \\
\hline 94 & Goosegrass & Eleusine indica & Poaceae & Rare & - \\
\hline 95 & Stinkgrass & Eragrostris cilianensis & Poaceae & Rare & 3 \\
\hline 96 & Woolly cupgrass & Eriochloa villosa & Poaceae & Common & - \\
\hline 97 & Tall fescue & Festuca arundinacea & Poaceae & Rare & - \\
\hline 98 & Wirestem muhly & Muhlenbergia frondosa & Poaceae & Common & - \\
\hline 99 & Witchgrass & Panicum capillare & Poaceae & Common & 3 \\
\hline 100 & Fall panicum & Panicum dichotomiflorum & Poaceae & Abundant & - \\
\hline 101 & Wild-proso millet & Panicum miliaceum & Poaceae & Rare & 3 \\
\hline 102 & Texas panicum & Panicum texanum & Poaceae & Rare & 1 \\
\hline 103 & Giant foxtail & Setaria faberi & Poaceae & Abundant & \\
\hline 104 & Yellow foxtail & Setaria glauca & Poaceae & Abundant & 3 \\
\hline 105 & Bristly foxtail & Setaria verticillata & Poaceae & Rare & - \\
\hline 106 & Green foxtail & Setaria viridis & Poaceae & Abundant & 3 \\
\hline 107 & Shattercane & Sorghum bicolor & Poaceae & Common & 3 \\
\hline 108 & Johnsongrass & Sorghum halepense & Poaceae & Common & 2 \\
\hline 109 & Corn & Zea mays & Poaceae & Abundant & \\
\hline 110 & Alpine smartweed & Polygenum viviparum & Polygonaceae & Rare & \\
\hline 111 & Swamp smartweed & Polygonит соссіпеит & Polygonaceae & Common & - \\
\hline 112 & Wild buckwheat & Polygonum convolvulus & Polygonaceae & Rare & 3 \\
\hline 113 & Pennsylvania smartweed & d Polygonum pennslyvanicum & Polygonaceae & Abundant & 2 \\
\hline 114 & Red sorrel & Rumex acetosella & Polygonaceae & Rare & - \\
\hline 115 & Pale dock & Rumex altissimus & Polygonaceae & Rare & - \\
\hline 116 & Curly dock & Rumex crispus & Polygonaceae & Rare & - \\
\hline
\end{tabular}


J.E. Losey et al.

\begin{tabular}{|c|c|c|c|c|c|}
\hline $\begin{array}{l}\text { Plant } \\
\text { Number }\end{array}$ & Plant Common Name & Plant Scientific Name & Plant Family & Abundance & $\begin{array}{c}\text { Weed } \\
\text { Reference }\end{array}$ \\
\hline 117 & Broadleaf dock & Rumex obtusifolius & Polygonaceae & Rare & - \\
\hline 118 & Common purslane & Portulaca oleracea & Portulaceae & Rare & 3 \\
\hline 119 & Rough cinquefoil & Potentilla norvegica & Rosaceae & Rare & - \\
\hline 120 & Sulphur cinquefoil & Potentilla recta & Rosaceae & Rare & - \\
\hline 121 & Wild blackberry & Rubus allegheniensis & Rosaceae & Rare & 4 \\
\hline 122 & Catchweed bed straw & Galium aparine & Rubiaceae & Rare & - \\
\hline 123 & Common mullein & Verbascum thapsus & Scrophulariaceae & Rare & - \\
\hline 124 & Field speedwell & Veronica agrestis & Scrophulariaceae & Rare & - \\
\hline 125 & Jimsonweed & Datura stramonium & Solanaceae & Common & \\
\hline 126 & Clammy groundcherry & Physalis heterophylla & Solanaceae & Common & - \\
\hline 127 & Virginia groundcherry & Physalis virginiana & Solanaceae & Common & - \\
\hline 128 & Horse nettle & Solanum carolinense & Solanaceae & Common & 1 \\
\hline 129 & Eastern blacknightshade & le Solanum ptycanthum & Solanaceae & Abundant & - \\
\hline 130 & Buffalo bur & Solanum rostratum & Solanaceae & Common & 2 \\
\hline 131 & Hairy nightshade & Solanum sarrachoides & Solanaceae & Rare & 3 \\
\hline 132 & Common cattail & Typha latifolia & Typhaceae & Rare & - \\
\hline
\end{tabular}

Weed codes are from the following references: 1 = Webster and Cobble, 1997; 2 = Wax et al., 1981; 3 = Schweizer et al., 1998; and 4 = Glenn et al., 1997.

Appendix 2.

Lepidopteran species reported to feed as larva on plants that occur in and around maize fields.

\begin{tabular}{|c|c|c|c|c|c|c|c|c|c|}
\hline Family & Species & Common name & $\mathrm{Jn}$ & $\mathrm{Jl}$ & $\mathrm{Ag}$ & $\begin{array}{l}\text { Host plant } \\
\text { code }^{1}\end{array}$ & $\begin{array}{l}\text { Plant } \\
\text { utilization } \\
\text { reference }^{2}\end{array}$ & $\begin{array}{l}\text { Global } \\
\text { rank }\end{array}$ & $\begin{array}{l}\text { National } \\
\text { rank }\end{array}$ \\
\hline Aegeridae & Albuna pyramidalis rubescens & - & - & - & - & 78 & 2 & - & - \\
\hline Agarastidae & Euscirrhopterus gloveri & _- & - & _- & _- & 118 & 13 & _ & _ \\
\hline Amatidae & Ctenucha virginica & virginia ctenucha & $\mathrm{Y}$ & Y & $\mathrm{N}$ & 93,99 & 13 & _- & _ \\
\hline Arctiidae & Apantensis figurata & _ & - & - & _ & 67 & 13 & _- & _ \\
\hline Arctiidae & Apantesis arge & _- & - & _- & _- & 81 & 13 & _- & _ \\
\hline Arctiidae & Apantesis ornata & _- & - & _- & _- & 50 & 13 & _- & _ \\
\hline Arctiidae & Apantesis parthenice & _- & $\mathrm{N}$ & $?$ & $\mathrm{Y}$ & 50 & 13 & _- & _ \\
\hline Arctiidae & Arachnis picta & painted arachnis & - & _- & _- & 67 & 13 & _- & _ \\
\hline Arctiidae & Arctia caja & great tiger moth & $\mathrm{Y}$ & $\mathrm{Y}$ & $\mathrm{Y}$ & 50 & 13 & G5 & _ \\
\hline Arctiidae & Estigmene acrea & saltmarsh moth & $\mathrm{Y}$ & $\mathrm{Y}$ & $\mathrm{Y}$ & 7 & 13 & G5 & _ \\
\hline Arctiidae & Euchaetias egle & $\begin{array}{l}\text { milkweed tussock } \\
\text { moth }\end{array}$ & $\mathrm{Y}$ & $\mathrm{Y}$ & $\mathrm{Y}$ & 11 & 10 & G5 & - \\
\hline Arctiidae & Haploa lecontei & Leconte's haploa & $\mathrm{Y}$ & $\mathrm{Y}$ & $\mathrm{Y}$ & 121 & 13 & G5 & - \\
\hline Arctiidae & Holomelina aurantiaca & - & $\mathrm{Y}$ & $\mathrm{Y}$ & $\mathrm{Y}$ & $31,50,109$ & 13 & G5 & - \\
\hline Arctiidae & Hyphantria cunea & fall webworm & $\mathrm{Y}$ & Y & $\mathrm{Y}$ & 67 & 14 & G5 & _ \\
\hline Arctiidae & Pyrrharctia isabella & banded woolybear & $\mathrm{Y}$ & $\mathrm{N}$ & $\mathrm{Y}$ & $82,25,121$ & 13 & - & - \\
\hline Arctiidae & Spilosoma vagans & _- & - & _- & _- & 50 & 13 & _ & _ \\
\hline Arctiidae & Spilosoma virginica & $\begin{array}{l}\text { yellow wolly bear } \\
\text { moth }\end{array}$ & $\mathrm{Y}$ & $\mathrm{N}$ & $\mathrm{Y}$ & $\begin{array}{l}16,25,50,58 \\
67,112,121 \\
130\end{array}$ & 13 & G5 & N5 \\
\hline Citheroniidae & Anisota senatoria & $\begin{array}{l}\text { orangestriped oak- } \\
\text { worm }\end{array}$ & $\mathrm{Y}$ & $\mathrm{Y}$ & $\mathrm{Y}$ & 121 & 13 & G5 & N5 \\
\hline Geometridae & Catopyrra coloraria & _- & $\mathrm{Y}$ & $\mathrm{Y}$ & $\mathrm{N}$ & 121 & 3 & G4 & _ \\
\hline Geometridae & Cepphis decoloraria & _ & $\mathrm{Y}$ & $\mathrm{Y}$ & $\mathrm{Y}$ & 121 & 3 & G5 & - \\
\hline
\end{tabular}


Lepidopteran species associated with maize

Appendix 2.

Continued.

\begin{tabular}{|c|c|c|c|c|c|c|c|c|c|}
\hline Family & Species & Common name & Jn & $\mathrm{J} 1$ & $\mathrm{Ag}$ & $\begin{array}{l}\text { Host plant } \\
\text { code }^{1}\end{array}$ & $\begin{array}{l}\text { Plant } \\
\text { utilization } \\
\text { reference }^{2}\end{array}$ & $\begin{array}{l}\text { Global } \\
\text { rank }\end{array}$ & $\begin{array}{l}\text { National } \\
\text { rank }\end{array}$ \\
\hline Geometridae & Chlorochlamys chloroleucaria & $\begin{array}{l}\text { blackberry looper } \\
\text { moth }\end{array}$ & $\mathrm{Y}$ & $\mathrm{Y}$ & $\mathrm{Y}$ & 121 & 13 & - & - \\
\hline Geometridae & Cingilia catenaria & chainspotted geometer & $\mathrm{N}$ & $\mathrm{N}$ & $\mathrm{N}$ & 53 & 13 & - & - \\
\hline Geometridae & Dysstroma citrata & dark marbled carpet & $\mathrm{N}$ & $\mathrm{Y}$ & $\mathrm{Y}$ & 121 & 13 & G5 & - \\
\hline Geometridae & Hydria undulata & scallop-shell moth & $\mathrm{N}$ & $\mathrm{Y}$ & $\mathrm{N}$ & 121 & 13 & - & - \\
\hline Geometridae & Phigalia titea & the half-wing & $\mathrm{N}$ & $\mathrm{N}$ & $\mathrm{N}$ & 121 & 13 & - & - \\
\hline Geometridae & Scopula inductata & soft-lined wave & $\mathrm{Y}$ & $\mathrm{Y}$ & $\mathrm{Y}$ & 16 & 13 & - & - \\
\hline Geometridae & Synchlora aerata & wavy-lined emerald & $\mathrm{Y}$ & $\mathrm{Y}$ & $\mathrm{Y}$ & 121 & 13 & G5 & - \\
\hline Geometridae & Xanthorhoe lacustrata & toothed brown carpet & $\mathrm{Y}$ & $\mathrm{Y}$ & $\mathrm{Y}$ & 121 & 13 & - & - \\
\hline Hesperiidae & Amblyscirtes hegon & pepper and salt skipper & $\mathrm{Y}$ & $\mathrm{Y}$ & $\mathrm{Y}$ & 107 & 13 & G5 & N5 \\
\hline Hesperiidae & Amblyscirtes nysa & nysa roadside skipper & $\mathrm{Y}$ & $\mathrm{Y}$ & $\mathrm{Y}$ & 106 & 5 & G5 & N5 \\
\hline Hesperiidae & Amblyscirtes vialis & roadside skipper & $\mathrm{Y}$ & $\mathrm{Y}$ & $\mathrm{Y}$ & 89 & 8 & G5 & N5 \\
\hline Hesperiidae & Atalopedes campestris & satchem & $\mathrm{Y}$ & $\mathrm{Y}$ & $\mathrm{Y}$ & 89 & 8 & G5 & N5 \\
\hline Hesperiidae & Carterocephalus palaemon & $\begin{array}{l}\text { checked skipper but- } \\
\text { terfly }\end{array}$ & $\mathrm{Y}$ & $\mathrm{Y}$ & $\mathrm{Y}$ & 82,92 & 13 & G5 & N5 \\
\hline Hesperiidae & Copaeodes minimus & southern skipperling & $\mathrm{Y}$ & $\mathrm{Y}$ & $\mathrm{Y}$ & 89 & 17 & G5 & N5 \\
\hline Hesperiidae & $\begin{array}{l}\text { Copaeodes minimus } \\
\text { aurantiaca }\end{array}$ & orange skipperling & $\mathrm{Y}$ & $\mathrm{Y}$ & $\mathrm{Y}$ & 92,89 & 17,13 & G5 & \\
\hline Hesperiidae & Erinnyis funeralis & funeral dusky wing & $\mathrm{Y}$ & $\mathrm{Y}$ & $\mathrm{Y}$ & 67 & 13 & G5 & N5 \\
\hline Hesperiidae & Erynnis lucilius & columbine dusky wing & $\mathrm{Y}$ & $\mathrm{Y}$ & $\mathrm{Y}$ & 78,50 & 17,13 & G4 & N4 \\
\hline Hesperiidae & Erynnis martialis & mottled duskywing & $\mathrm{Y}$ & $\mathrm{Y}$ & $\mathrm{Y}$ & 5,6 & 13 & G3G4 & $\mathrm{N} 3 \mathrm{~N} 4$ \\
\hline Hesperiidae & Hesperia sassacus & indian skipper & $\mathrm{Y}$ & $\mathrm{Y}$ & $\mathrm{N}$ & 92 & 5 & G5 & N5 \\
\hline Hesperiidae & Hylephila phyleus & fiery skipper & $\mathrm{Y}$ & $\mathrm{Y}$ & $\mathrm{Y}$ & 89 & 9 & G5 & N5 \\
\hline Hesperiidae & Lerema accius & clouded skipper & $\mathrm{Y}$ & $\mathrm{Y}$ & $\mathrm{Y}$ & 99,109 & 11,9 & G5 & N5 \\
\hline Hesperiidae & Lerodea eufala & eufala skipper & $\mathrm{Y}$ & $\mathrm{Y}$ & $\mathrm{Y}$ & 108 & 5 & G5 & N5 \\
\hline Hesperiidae & Panoquina errans & wandering skipper & $\mathrm{N}$ & $\mathrm{Y}$ & $\mathrm{Y}$ & 89 & 7 & - & - \\
\hline Hesperiidae & Pholisora catullus & common sooty wing & $\mathrm{Y}$ & $\mathrm{Y}$ & $\mathrm{Y}$ & $3,50,5$ & $6,9,5$ & G5 & N5 \\
\hline Hesperiidae & Poanes melane & umber skipper & $\mathrm{Y}$ & $\mathrm{Y}$ & $\mathrm{Y}$ & 89,91 & 13 & G5 & N5 \\
\hline Hesperiidae & Polites mystic & long dash & $\mathrm{Y}$ & $\mathrm{Y}$ & $\mathrm{Y}$ & 82 & 11 & G5 & N5 \\
\hline Hesperiidae & Polites sabuleti & sandhill skipper & - & - & - & 89 & 13 & G5 & N5 \\
\hline Hesperiidae & Polites themistocles & tawny edged skipper & $\mathrm{Y}$ & $\mathrm{Y}$ & $\mathrm{Y}$ & 82 & 13 & G5 & N5 \\
\hline Hesperiidae & Polites vibex & whirlabout & $\mathrm{Y}$ & $\mathrm{Y}$ & $\mathrm{Y}$ & 89 & 8 & G5 & N5 \\
\hline Hesperiidae & Pyrgus communis & checkered skipper & $\mathrm{Y}$ & $\mathrm{Y}$ & $\mathrm{Y}$ & 50,73 & 8,11 & G5 & N5 \\
\hline Hesperiidae & Pyrgus syrichtus & $\begin{array}{l}\text { tropical checkered } \\
\text { skipper }\end{array}$ & $\mathrm{Y}$ & $\mathrm{Y}$ & $\mathrm{Y}$ & 74 & 9 & G5 & - \\
\hline Hesperiidae & Staphylus ceos & $\begin{array}{l}\text { golden-headed sooty- } \\
\text { wing }\end{array}$ & $\mathrm{Y}$ & $\mathrm{Y}$ & $\mathrm{Y}$ & 51 & 7 & G5 & N4 \\
\hline Hesperiidae & Staphylus hayhurstii & southern sooty wing & $\mathrm{Y}$ & $\mathrm{Y}$ & $\mathrm{Y}$ & 50 & 8 & G5 & N5 \\
\hline Hesperiidae & Staphylus mazans & $\begin{array}{l}\text { southern scalloped } \\
\text { sooty wing }\end{array}$ & $\mathrm{Y}$ & $\mathrm{Y}$ & $\mathrm{Y}$ & 5 & 5 & G5 & N3N4 \\
\hline Hesperiidae & Thorybes bathyllus & southern cloudy wing & $\mathrm{N}$ & $\mathrm{N}$ & $\mathrm{N}$ & 68 & 17 & G5 & N5 \\
\hline Hesperiidae & Thorybes pylades & northern cloudy wing & $\mathrm{Y}$ & $\mathrm{Y}$ & $\mathrm{Y}$ & 67,68 & 8,13 & G5 & N5 \\
\hline
\end{tabular}


J.E. Losey et al.

Appendix 2.

Continued.

\begin{tabular}{|c|c|c|c|c|c|c|c|c|c|}
\hline Family & Species & Common name & $\mathrm{Jn}$ & $\mathrm{J} 1$ & $\mathrm{Ag}$ & $\begin{array}{l}\text { Host plant } \\
\text { code }^{1}\end{array}$ & $\begin{array}{l}\text { Plant } \\
\text { utilization } \\
\text { reference }\end{array}$ & $\begin{array}{l}\text { Global } \\
\text { rank }\end{array}$ & $\begin{array}{l}\text { National } \\
\text { rank }\end{array}$ \\
\hline Hesperiidae & Urbanus simplicius & plain longtail & - & - & - & 89 & 7 & G5 & - \\
\hline Hesperiidae & Wallengrenia otho & - & - & - & - & 92 & 13 & G5 & - \\
\hline Hesperiidae & Wallengrenia otho egeremet & broken-dash & - & - & - & 92 & 1 & G5 & N5 \\
\hline $\begin{array}{l}\text { Lasiocampi- } \\
\text { dae }\end{array}$ & Malacosoma californica & western tent caterpillar & $\mathrm{Y}$ & $\mathrm{Y}$ & $\mathrm{Y}$ & 121 & 13 & - & - \\
\hline Liparidae & Nygmia phaeorrhoea & brown-tail moth & $?$ & $?$ & $?$ & 121 & 13 & - & - \\
\hline Liparidae & Orgyia leucostigma & - & $?$ & $?$ & $?$ & 33,50 & 13 & G5 & - \\
\hline Lycaenidae & Brephidium exile & western pigmy blue & $\mathrm{Y}$ & $\mathrm{Y}$ & $\mathrm{Y}$ & 50,5 & 5,9 & G5 & N5 \\
\hline Lycaenidae & Everes comyntas & eastern tailed blue & $\mathrm{Y}$ & $\mathrm{Y}$ & $\mathrm{Y}$ & 68 & 5 & - & - \\
\hline Lycaenidae & Everes comyntas & eastern tailed blue & $\mathrm{Y}$ & $\mathrm{Y}$ & $\mathrm{Y}$ & 67,16 & 8,13 & G5 & N5 \\
\hline Lycaenidae & Gaeides xanthoides & great grey copper & $\mathrm{Y}$ & $\mathrm{Y}$ & $\mathrm{Y}$ & 116,117 & 9 & - & - \\
\hline Lycaenidae & Hemiargus gyas & - & - & - & - & 67 & 13 & - & - \\
\hline Lycaenidae & Leptotes marina & marine blue & - & - & - & 67 & 13 & G5 & N5 \\
\hline Lycaenidae & Lycaeides melissa & orange-bordered blue & $\mathrm{Y}$ & $\mathrm{Y}$ & $\mathrm{Y}$ & 67 & 9 & G5 & N5 \\
\hline Lycaenidae & Lycaeides melissa samuelis & karner blue & $\mathrm{Y}$ & $\mathrm{Y}$ & $\mathrm{N}$ & 67 & 12 & G5 & $\mathrm{N} 2$ \\
\hline Lycaenidae & Lycaena helloides & purplish copper & $\mathrm{Y}$ & $\mathrm{Y}$ & $\mathrm{Y}$ & 114 & 17 & G5 & N5 \\
\hline Lycaenidae & Lycaena hyllus & bronze copper & $\mathrm{Y}$ & $\mathrm{Y}$ & $\mathrm{Y}$ & 116 & 11 & G5 & N5 \\
\hline Lycaenidae & Lycaena phlaeas & American copper & $\mathrm{Y}$ & $\mathrm{Y}$ & $\mathrm{Y}$ & 114,116 & 11 & G5 & N5 \\
\hline Lycaenidae & Plebejus saepiolus & greenish blue & $\mathrm{Y}$ & $\mathrm{Y}$ & $\mathrm{Y}$ & 115,116 & 5 & G5 & N5 \\
\hline Noctuidae & Acronicta impressa & - & - & - & - & 121 & 13 & - & - \\
\hline Noctuidae & Acronicta longa & $\begin{array}{l}\text { long-winged dagger } \\
\text { moth }\end{array}$ & $\mathrm{Y}$ & $\mathrm{Y}$ & $\mathrm{Y}$ & 121 & 13 & - & - \\
\hline Noctuidae & Acronicta oblinita & smeared dagger moth & $\mathrm{Y}$ & $\mathrm{Y}$ & $\mathrm{Y}$ & 121,132 & 13 & G5 & N5 \\
\hline Noctuidae & Acronicta spingera & $\begin{array}{l}\text { nondescript dagger } \\
\text { moth }\end{array}$ & $\mathrm{Y}$ & $\mathrm{Y}$ & $\mathrm{Y}$ & 121 & 13 & G4 & - \\
\hline Noctuidae & Actebia fennica & black army cutworm & $\mathrm{N}$ & $\mathrm{Y}$ & $\mathrm{Y}$ & 121,123 & 13 & - & - \\
\hline Noctuidae & Agrotis gladiaria & swordsman dart & $\mathrm{N}$ & $\mathrm{N}$ & $?$ & 121 & 13 & G5 & - \\
\hline Noctuidae & Agrotis ipsilon & black cutworm & $\mathrm{Y}$ & $\mathrm{Y}$ & $\mathrm{Y}$ & 37 & 13 & G5 & - \\
\hline Noctuidae & Agrotis orthogonia & pale western cutworm & - & - & - & $20,37,50$ & 13 & - & - \\
\hline Noctuidae & Agrotis vetusta & old man dart & $\mathrm{N}$ & $\mathrm{Y}$ & $\mathrm{Y}$ & 123 & 13 & - & - \\
\hline Noctuidae & Anicla infecta & $\begin{array}{l}\text { green clover worm } \\
\text { moth }\end{array}$ & $\mathrm{Y}$ & $\mathrm{Y}$ & $\mathrm{Y}$ & 92 & 13 & G5 & - \\
\hline Noctuidae & Anomis erosa & yellow scallop moth & $\mathrm{N}$ & $\mathrm{N}$ & $\mathrm{Y}$ & 72 & 13 & G5 & - \\
\hline Noctuidae & Anticarsia gemmatilis & velvetbean caterpillar & $\mathrm{N}$ & $\mathrm{N}$ & $\mathrm{N}$ & 67 & 13 & G5 & - \\
\hline Noctuidae & Archanara oblonga & $\begin{array}{l}\text { oblong sedge borer } \\
\text { moth }\end{array}$ & $\mathrm{N}$ & $\mathrm{Y}$ & $\mathrm{Y}$ & 132 & 13 & G5 & - \\
\hline Noctuidae & Autographa californica & alfalfa looper & - & - & - & 67 & 13 & - & - \\
\hline Noctuidae & Autographa precationis & common looper moth & $\mathrm{Y}$ & $\mathrm{Y}$ & Y & 50 & 13 & G5 & - \\
\hline Noctuidae & Bellura densa & - & - & - & - & 132 & 13 & - & - \\
\hline Noctuidae & Bellura obliqua & - & - & - & - & 132 & 13 & - & - \\
\hline Noctuidae & Caenurgina erechtea & forage looper & $\mathrm{Y}$ & $\mathrm{Y}$ & Y & 16,67 & 13 & G5 & - \\
\hline Noctuidae & Cercyonis latipes & small mocis & $\mathrm{Y}$ & $\mathrm{Y}$ & $\mathrm{Y}$ & 92,109 & 13 & - & _ \\
\hline
\end{tabular}


Lepidopteran species associated with maize

Appendix 2.

Continued.

\begin{tabular}{|c|c|c|c|c|c|c|c|c|c|}
\hline Family & Species & Common name & Jn & $\mathrm{J} 1$ & $\mathrm{Ag}$ & $\begin{array}{l}\text { Host plant } \\
\text { code }^{\mathrm{I}}\end{array}$ & $\begin{array}{l}\text { Plant } \\
\text { utilization } \\
\text { reference }^{2}\end{array}$ & $\begin{array}{l}\text { Global } \\
\text { rank }\end{array}$ & $\begin{array}{l}\text { National } \\
\text { rank }\end{array}$ \\
\hline Noctuidae & Copablepharon viridisparsum & - & - & - & - & 37 & 13 & - & - \\
\hline Noctuidae & Crymodes devastator & glassy cutworm moth & $\mathrm{Y}$ & $\mathrm{Y}$ & $\mathrm{Y}$ & 67 & 13 & - & - \\
\hline Noctuidae & Dargida procincta & - & - & - & - & 82 & 13 & - & - \\
\hline Noctuidae & Erastria carneola & - & $\mathrm{Y}$ & $\mathrm{Y}$ & $\mathrm{Y}$ & 116 & 13 & - & - \\
\hline Noctuidae & Euxoa auxiliaris & army cutworm & - & - & - & 37 & 13 & - & - \\
\hline Noctuidae & Euxoa costata & - & - & - & - & 53 & 13 & - & - \\
\hline Noctuidae & Euxoa divergens & divergent dart & $\mathrm{Y}$ & $\mathrm{Y}$ & $\mathrm{N}$ & 67 & 13 & G4 & - \\
\hline Noctuidae & Euxoa messoria & reaper dart & $\mathrm{N}$ & $\mathrm{N}$ & $\mathrm{Y}$ & 25 & 16 & _ & _- \\
\hline Noctuidae & Euxoa pallipennis & - & - & - & - & 53 & 13 & - & - \\
\hline Noctuidae & Euxoa pleuritica & - & $?$ & $?$ & $?$ & 118 & 3 & G4 & - \\
\hline Noctuidae & Euxoa scandens & white cutworm & $\mathrm{Y}$ & $\mathrm{Y}$ & $\mathrm{N}$ & 121 & 13 & G5 & - \\
\hline Noctuidae & Euxoa tristicula & - & $\mathrm{N}$ & $\mathrm{Y}$ & $\mathrm{N}$ & 121 & 3 & G4 & - \\
\hline Noctuidae & Feltia subterranea & granulate cutworm & $\mathrm{N}$ & $\mathrm{Y}$ & $\mathrm{Y}$ & 31,6 & 15,13 & - & - \\
\hline Noctuidae & Heliothis obsoleta & cotton boll-worm & $?$ & $?$ & $\mathrm{Y}$ & $\begin{array}{l}32,50,58,67 \\
73,91,107\end{array}$ & 13 & - & - \\
\hline Noctuidae & Heliothis phloxiphagus & - & - & - & - & 67 & 13 & G5 & - \\
\hline Noctuidae & Heliothis virescens & tobacco budworm & $\mathrm{Y}$ & $\mathrm{Y}$ & $\mathrm{Y}$ & 67,116 & 13 & G5 & - \\
\hline Noctuidae & Hyppa xylinoides & common hyppa & $\mathrm{Y}$ & $\mathrm{Y}$ & $\mathrm{Y}$ & 31,50 & 13 & - & - \\
\hline Noctuidae & Lacinipolia lorea & bridled arches & $\mathrm{Y}$ & $\mathrm{Y}$ & $\mathrm{Y}$ & 67 & 13 & - & - \\
\hline Noctuidae & Lacinipolia olivacea & olive arches & $\mathrm{y}$ & $\mathrm{Y}$ & $\mathrm{Y}$ & 81 & 13 & - & - \\
\hline Noctuidae & Lacinipolia renigera & bristly cutworm & $\mathrm{Y}$ & $\mathrm{Y}$ & $\mathrm{Y}$ & 67,81 & 13 & G5 & - \\
\hline Noctuidae & Leucania latiuscula & - & - & - & _ & 107,109 & 13 & _ & - \\
\hline Noctuidae & Leucania multilinea & many-lined wainscot & $\mathrm{Y}$ & $\mathrm{Y}$ & $\mathrm{Y}$ & 107 & 13 & G5 & _- \\
\hline Noctuidae & Leucania pseudargyria guenee & false wainscot & $\mathrm{Y}$ & $\mathrm{Y}$ & $\mathrm{Y}$ & 132 & 13 & G5 & - \\
\hline Noctuidae & Leuconycta diphteroides & green leuconycta & $\mathrm{Y}$ & $\mathrm{Y}$ & Y & 29 & 10 & G5 & - \\
\hline Noctuidae & Luperina stipata & - & - & - & - & 104 & 13 & G4 & - \\
\hline Noctuidae & Mamestra configurata & bertha armyworm & - & - & - & 67 & 13 & - & - \\
\hline Noctuidae & Melanchra picta & painted mamestra & - & - & - & $8,9,67$ & 13 & G4 & - \\
\hline Noctuidae & Nola sorghiella & - & $\mathrm{Y}$ & $\mathrm{Y}$ & $\mathrm{Y}$ & 107 & 13 & - & - \\
\hline Noctuidae & Ogdoconta cinereola & common pinkband & $\mathrm{Y}$ & $\mathrm{Y}$ & $\mathrm{Y}$ & $16,13,25$ & 16,3 & G5 & - \\
\hline Noctuidae & Palthis angulais & dark-spotted palthis & $\mathrm{Y}$ & $\mathrm{Y}$ & $\mathrm{Y}$ & 121 & 13 & G5 & - \\
\hline Noctuidae & Papaiema nebris & stalk borer & $\mathrm{N}$ & $\mathrm{N}$ & $\mathrm{N}$ & 123 & 13 & G5 & N5 \\
\hline Noctuidae & Papaipema arctivorens & - & - & - & - & 20 & 13 & G5 & N5 \\
\hline Noctuidae & $\begin{array}{l}\text { Papaipema arctivorens } \\
\text { hampson }\end{array}$ & - & - & - & - & 21 & 13 & - & - \\
\hline Noctuidae & Papaipema cataphracta & burdock borer & $\mathrm{N}$ & $\mathrm{N}$ & $\mathrm{N}$ & 109,121 & 13 & G5 & N5 \\
\hline Noctuidae & Papaipema nebris & stalk borer & $\mathrm{N}$ & $\mathrm{N}$ & $\mathrm{N}$ & $\begin{array}{l}13,16,20,32 \\
50,121\end{array}$ & 13 & G5 & N5 \\
\hline Noctuidae & Papaipema necopina & - & - & - & - & 26 & 13 & G4 & N4 \\
\hline Noctuidae & Papaipema rutila & - & - & - & - & 16 & 13 & G4 & N4 \\
\hline Noctuidae & Peridroma margaritosa & pearly underwing & $\mathrm{Y}$ & $\mathrm{Y}$ & $\mathrm{Y}$ & $\begin{array}{l}7,81,109,118 \\
121,125\end{array}$ & 13 & - & - \\
\hline
\end{tabular}


J.E. Losey et al.

Appendix 2.

Continued.

\begin{tabular}{|c|c|c|c|c|c|c|c|c|c|}
\hline Family & Species & Common name & Jn & $\mathrm{J} 1$ & $\mathrm{Ag}$ & $\begin{array}{l}\text { Host plant } \\
\text { code }^{1}\end{array}$ & $\begin{array}{l}\text { Plant } \\
\text { utilization } \\
\text { reference }\end{array}$ & $\begin{array}{l}\text { Global } \\
\text { rank }\end{array}$ & $\begin{array}{l}\text { National } \\
\text { rank }\end{array}$ \\
\hline Noctuidae & Phosphila turbulenta & turbulent phosphila & $\mathrm{Y}$ & $\mathrm{Y}$ & $\mathrm{Y}$ & 128 & 13 & G5 & - \\
\hline Noctuidae & Plagiomimicus pityochromus & black-barred brown & $\mathrm{N}$ & $\mathrm{N}$ & $\mathrm{Y}$ & 16 & 13 & _ & - \\
\hline Noctuidae & Plathypena scabra & $\begin{array}{l}\text { green clover worm } \\
\text { moth }\end{array}$ & $\mathrm{Y}$ & $\mathrm{Y}$ & $?$ & $67,8,16,91$ & 10,13 & G5 & - \\
\hline Noctuidae & Polia atlantica & - & - & - & - & 67 & 13 & - & - \\
\hline Noctuidae & Polia grandis & _ & $\mathrm{Y}$ & $\mathrm{Y}$ & $\mathrm{Y}$ & 50 & 13 & - & - \\
\hline Noctuidae & Polia legitima & $\begin{array}{l}\text { striped garden cater- } \\
\text { pillar }\end{array}$ & $\mathrm{Y}$ & $\mathrm{Y}$ & $\mathrm{Y}$ & 92,121 & 13 & - & - \\
\hline Noctuidae & Protorthodes incincta & _ & - & - & - & 67 & 13 & - & - \\
\hline Noctuidae & Pseudaletia unipuncta & white-speck & $\mathrm{Y}$ & $\mathrm{Y}$ & $\mathrm{Y}$ & $\begin{array}{l}6,16,82,89 \\
92,101,107\end{array}$ & 13 & G5 & N5 \\
\hline Noctuidae & Pseudorthordes irrorata & - & - & - & - & 81 & 13 & - & - \\
\hline Noctuidae & Psychomorpha epimenis & grapevine epimenis & $\mathrm{Y}$ & $\mathrm{N}$ & $\mathrm{N}$ & 33 & 13 & G5 & - \\
\hline Noctuidae & Pyrrhia umbra & bordered sallow & $\mathrm{Y}$ & $\mathrm{Y}$ & $\mathrm{Y}$ & 113,121 & 13 & - & - \\
\hline Noctuidae & Schinia florida & primrose moth & $\mathrm{Y}$ & $\mathrm{Y}$ & $\mathrm{Y}$ & 78 & 4 & _- & _- \\
\hline Noctuidae & Schinia marginata & _- & - & - & - & 13 & 13 & _- & - \\
\hline Noctuidae & Schinia rivulosa & _- & $\mathrm{Y}$ & Y & $\mathrm{Y}$ & 13 & 4 & G5 & _- \\
\hline Noctuidae & Schinia thoreaui & Thoreau's flower moth & $\mathrm{Y}$ & $\mathrm{Y}$ & Y & 16 & 13 & - & - \\
\hline Noctuidae & Scotogramma trifolii & clover cutworm & $\mathrm{Y}$ & $\mathrm{Y}$ & $\mathrm{Y}$ & 50,118 & 13 & - & - \\
\hline Noctuidae & Simyra henrici & Henry's marsh moth & $\mathrm{Y}$ & $\mathrm{Y}$ & Y & 132 & 13 & G5 & - \\
\hline Noctuidae & Spodoptera dolichos & - & $\mathrm{Y}$ & $\mathrm{N}$ & $\mathrm{Y}$ & 125 & 3 & G5 & - \\
\hline Noctuidae & Spodoptera eridania & southern army worm & - & - & - & $7,91,92$ & 13 & G5 & - \\
\hline Noctuidae & Spodoptera exigua & beet army worm & - & - & - & $5,25,50,67$ & 13 & G5 & - \\
\hline Noctuidae & Spodoptera frugiperda & fall army worm & $\mathrm{N}$ & $\mathrm{Y}$ & $\mathrm{Y}$ & $\begin{array}{l}5,32,50,56 \\
62,67,91,92 \\
101,102,107\end{array}$ & 13 & G5 & - \\
\hline Noctuidae & spodoptera ornithogalli & cotton cutworm & - & - & - & $\begin{array}{l}5,7,22,50,58 \\
67,81,121 \\
125,128\end{array}$ & 13 & G5 & - \\
\hline Noctuidae & Spodoptera praefica & $\begin{array}{l}\text { western yellowstriped } \\
\text { armyworm }\end{array}$ & - & - & - & $\begin{array}{l}50,58,67,107, \\
121\end{array}$ & 13 & - & - \\
\hline Noctuidae & Stibadium spumosum & frothy moth & $\mathrm{N}$ & $\mathrm{N}$ & $\mathrm{Y}$ & 25 & 3 & - & - \\
\hline Noctuidae & Tarachidia candefacta & $\begin{array}{l}\text { olive-shaded bird- } \\
\text { dropping moth }\end{array}$ & $\mathrm{Y}$ & $\mathrm{Y}$ & $\mathrm{Y}$ & 13 & 13 & G5 & - \\
\hline Noctuidae & Tarachidia erastriodes & $\begin{array}{l}\text { small bird-dropping } \\
\text { moth }\end{array}$ & $\mathrm{Y}$ & $\mathrm{Y}$ & $\mathrm{Y}$ & 13 & 13 & G5 & - \\
\hline Noctuidae & Trichoplusia ni & cabagge looper & - & - & - & 37,50 & 13 & - & - \\
\hline Notodontidae & Datana ministra & $\begin{array}{l}\text { yellownecked } \\
\text { caterpillar }\end{array}$ & $\mathrm{Y}$ & $\mathrm{N}$ & $\mathrm{N}$ & 121 & 13 & G5 & - \\
\hline Notodontidae & Heterocampa guttivitta & maple prominent & $\mathrm{Y}$ & $\mathrm{Y}$ & $\mathrm{Y}$ & 121 & 13 & G5 & - \\
\hline Notodontidae & Schizura concinna & $\begin{array}{l}\text { red-humped } \\
\text { caterpillar moth }\end{array}$ & $\mathrm{Y}$ & $\mathrm{Y}$ & $\mathrm{Y}$ & 121 & 13 & - & - \\
\hline Notodontidae & Schizura ipomoeae & $\begin{array}{l}\text { morning-glory } \\
\text { prominent }\end{array}$ & $\mathrm{Y}$ & $\mathrm{Y}$ & $\mathrm{Y}$ & 56,121 & 13 & G5 & - \\
\hline
\end{tabular}


Lepidopteran species associated with maize

Appendix 2.

Continued.

\begin{tabular}{|c|c|c|c|c|c|c|c|c|c|}
\hline Family & Species & Common name & Jn & $\mathrm{J} 1$ & $\mathrm{Ag}$ & $\begin{array}{l}\text { Host plant } \\
\text { code }^{1}\end{array}$ & $\begin{array}{l}\text { Plant } \\
\text { utilization } \\
\text { reference }^{2}\end{array}$ & $\begin{array}{l}\text { Global } \\
\text { rank }\end{array}$ & $\begin{array}{l}\text { National } \\
\text { rank }\end{array}$ \\
\hline Notodontidae & Schizura unicornis & $\begin{array}{l}\text { unicorn caterpillar } \\
\text { moth }\end{array}$ & $\mathrm{Y}$ & $\mathrm{Y}$ & $\mathrm{Y}$ & 121 & 13 & - & - \\
\hline Nymphalidae & Charidryas gorgone & gorgone crescentspot & $\mathrm{Y}$ & $\mathrm{Y}$ & $\mathrm{N}$ & 16 & 9 & _- & _- \\
\hline Nymphalidae & Charidryas nycteis & silvery checkerspot & _- & - & _- & 22 & 13 & G5 & _- \\
\hline Nymphalidae & Chlosyne gorgone & $\begin{array}{l}\text { great plains } \\
\text { checkerspot }\end{array}$ & $\mathrm{Y}$ & $\mathrm{Y}$ & $\mathrm{Y}$ & 25 & 11 & G5 & N5 \\
\hline Nymphalidae & Chlosyne lacinia & bordered patch & $\mathrm{Y}$ & $\mathrm{Y}$ & $\mathrm{Y}$ & 25,16 & 10,7 & G5 & N5 \\
\hline Nymphalidae & Chlosyne lacinia adjutrix & bordered patch & $\mathrm{Y}$ & $\mathrm{Y}$ & $\mathrm{Y}$ & 16 & 5 & _- & _ \\
\hline Nymphalidae & Chlosyne nycteis & $\begin{array}{l}\text { streamside } \\
\text { checkerspot }\end{array}$ & $\mathrm{Y}$ & $\mathrm{Y}$ & $\mathrm{Y}$ & 25 & 11 & G5 & N5 \\
\hline Nymphalidae & Chlosyne palla & northern checkerspot & $\mathrm{Y}$ & $\mathrm{Y}$ & $\mathrm{Y}$ & 23 & 7 & G5 & N5 \\
\hline Nymphalidae & Chlosyne whitneyi & rockslide checkerspot & $?$ & $\mathrm{Y}$ & $\mathrm{Y}$ & 23 & 7 & G4G5 & N4 \\
\hline Nymphalidae & Cyllopsis gemma & gemmed satyr & $\mathrm{Y}$ & $\mathrm{Y}$ & $\mathrm{Y}$ & 89 & 8 & G5 & N5 \\
\hline Nymphalidae & Danaus gilippus & queen butterfly & $\mathrm{N}$ & $\mathrm{Y}$ & $\mathrm{Y}$ & 11 & 17 & G5 & N5 \\
\hline Nymphalidae & Danaus plexippus & monarch butterfly & $\mathrm{Y}$ & $\mathrm{Y}$ & $\mathrm{Y}$ & 11 & 7 & G4 & $\mathrm{N} 2 \mathrm{~N} 3$ \\
\hline Nymphalidae & Euphydryas gillettii & $\begin{array}{l}\text { yellowstone } \\
\text { checkerspot }\end{array}$ & $\mathrm{Y}$ & $\mathrm{Y}$ & $\mathrm{Y}$ & 124 & 7 & - & - \\
\hline Nymphalidae & Euphydryas phaeton & the baltimore & $\mathrm{Y}$ & $\mathrm{Y}$ & $\mathrm{Y}$ & 132 & 13 & G4 & N4 \\
\hline Nymphalidae & Euptoieta claudia & variegated frittilary & $\mathrm{Y}$ & $\mathrm{Y}$ & $\mathrm{Y}$ & 118 & 11 & G5 & N5 \\
\hline Nymphalidae & Junonia coenia & common buckeye & $\mathrm{Y}$ & $\mathrm{Y}$ & $\mathrm{Y}$ & 81 & 7 & _- & _- \\
\hline Nymphalidae & Megisto rubricata & red satyr & $\mathrm{Y}$ & $\mathrm{Y}$ & $\mathrm{Y}$ & 89 & 17 & G5 & N5 \\
\hline Nymphalidae & Nymphalis californica & california tortoise shell & $\mathrm{Y}$ & $\mathrm{Y}$ & $\mathrm{Y}$ & 67 & 13 & G5 & N5 \\
\hline Nymphalidae & Nymphalis milberti & Milbert's tortoise shell & $\mathrm{N}$ & $\mathrm{Y}$ & $\mathrm{Y}$ & 25 & 13 & G5 & N5 \\
\hline Nymphalidae & Occidryas taylori & - & - & - & - & 81 & 13 & - & - \\
\hline Nymphalidae & Phyciodes pictus & painted crescent & $\mathrm{Y}$ & $\mathrm{Y}$ & $\mathrm{Y}$ & 67,55 & 13,17 & G5 & - \\
\hline Nymphalidae & Phyciodes tharos & pearl crescent & $\mathrm{Y}$ & $\mathrm{Y}$ & $\mathrm{Y}$ & 23 & 7 & G5 & N5 \\
\hline Nymphalidae & Polygonia comma & comma butterfly & - & - & - & 13,16 & 13 & G5 & N5 \\
\hline Nymphalidae & Polygonia gracilis & graceful angle wing & - & - & - & 16 & 13 & G5 & N5 \\
\hline Nymphalidae & Polygonia interrogationis & question mark & - & - & - & 13,16 & 13 & G5 & N5 \\
\hline Nymphalidae & Proclossiana eunomia & - & - & - & - & 110 & 5 & G5 & \\
\hline Nymphalidae & Proclossiana titania & - & _ & - & _ & 110 & 17 & _ & _- \\
\hline Nymphalidae & Vanessa atalanta & red admiral, alderman & $\mathrm{Y}$ & $\mathrm{Y}$ & $\mathrm{Y}$ & 13,16 & 13 & G5 & N5 \\
\hline Nymphalidae & Vanessa cardui & painted lady & $\mathrm{Y}$ & $\mathrm{Y}$ & $\mathrm{Y}$ & $\begin{array}{l}18,21,20,50, \\
74\end{array}$ & 16,11 & G5 & N5 \\
\hline Nymphalidae & Vanessa virginiensis & painted beauty & $\mathrm{Y}$ & $\mathrm{Y}$ & $\mathrm{Y}$ & 20 & 13 & G5 & N5 \\
\hline Papilionidae & Battus philenor & pipevine swallowtail & $\mathrm{Y}$ & $\mathrm{Y}$ & $\mathrm{Y}$ & 112 & 11 & G5 & N5 \\
\hline Papilionidae & Papilio polyxenes & black swallowtail & $\mathrm{Y}$ & $\mathrm{Y}$ & $\mathrm{Y}$ & 8 & 9 & G5 & N5 \\
\hline Papilionidae & Papilio zelicaon lucas & anise swallowtail & $\mathrm{Y}$ & $\mathrm{Y}$ & $\mathrm{N}$ & 8 & 13 & G5 & N5 \\
\hline Pieridae & Anthocharis cethura & desert orangetip & $\mathrm{N}$ & $\mathrm{N}$ & $\mathrm{N}$ & 36 & 13 & G4G5 & N4N5 \\
\hline Pieridae & Anthocharis midea & falcate orangetip & $\mathrm{N}$ & $\mathrm{N}$ & $\mathrm{N}$ & 34 & 9 & G4G5 & N4N5 \\
\hline Pieridae & Anthocharis sara stella & western orange tip & $\mathrm{Y}$ & $\mathrm{Y}$ & $\mathrm{N}$ & 37 & 13 & - & - \\
\hline Pieridae & Artogeia napi & sharp-veined white & $\mathrm{Y}$ & $\mathrm{Y}$ & $\mathrm{Y}$ & 37 & 13 & G5 & _- \\
\hline
\end{tabular}


J.E. Losey et al.

Appendix 2.

Continued.

\begin{tabular}{|c|c|c|c|c|c|c|c|c|c|}
\hline Family & Species & Common name & Jn & $\mathrm{J} 1$ & $\mathrm{Ag}$ & $\begin{array}{l}\text { Host plant } \\
\text { code }^{1}\end{array}$ & $\begin{array}{l}\text { Plant } \\
\text { utilization } \\
\text { reference }^{2}\end{array}$ & $\begin{array}{l}\text { Global } \\
\text { rank }\end{array}$ & $\begin{array}{l}\text { National } \\
\text { rank }\end{array}$ \\
\hline Pieridae & Colias alexandra & $\begin{array}{l}\text { queen alexandra's sul- } \\
\text { phur }\end{array}$ & $\mathrm{Y}$ & $\mathrm{Y}$ & $\mathrm{Y}$ & 68 & 17 & G5 & N5 \\
\hline Pieridae & Colias eurytheme & alphalfa sulphur & $\mathrm{Y}$ & $\mathrm{Y}$ & $\mathrm{Y}$ & 67 & 13 & G5 & N5 \\
\hline Pieridae & Colias philodice & clouded sulphur & $\mathrm{Y}$ & $\mathrm{Y}$ & $?$ & 68,67 & 17,5 & G5 & N5 \\
\hline Pieridae & Falcapoca midea & falcate orangetip & - & - & - & 34,38 & 6 & - & - \\
\hline Pieridae & Nathalis iole & dainty sulphur & $\mathrm{Y}$ & $\mathrm{Y}$ & $\mathrm{Y}$ & 48 & 6 & G5 & N5 \\
\hline Pieridae & Pieris rapae & $\begin{array}{l}\text { imported } \\
\text { cabbageworm }\end{array}$ & $\mathrm{Y}$ & $\mathrm{Y}$ & $\mathrm{Y}$ & 37 & 13 & G5 & $\mathrm{Ne}$ \\
\hline Pieridae & Pontia beckerii & sagebrush white & $\mathrm{Y}$ & $\mathrm{N}$ & $\mathrm{Y}$ & 37 & 9 & G5 & N5 \\
\hline Pieridae & Pontia occidentalis & western white & $\mathrm{Y}$ & $\mathrm{Y}$ & $\mathrm{Y}$ & 37 & 7 & G5 & N5 \\
\hline Pieridae & Pontra protodice & checkered white & $\mathrm{Y}$ & $\mathrm{Y}$ & $\mathrm{Y}$ & $22,36,38,128$ & 5,13 & - & - \\
\hline Pieridae & Zerene cesonia & southern dogface & $\mathrm{Y}$ & $\mathrm{Y}$ & $\mathrm{Y}$ & 67 & 8 & G5 & N5 \\
\hline Saturniidae & Automeris io & io moth & $\mathrm{Y}$ & $\mathrm{Y}$ & $\mathrm{Y}$ & 121 & 13 & G5 & N5 \\
\hline Saturniidae & Hemileuca nevadensis stretch & Nevada buck moth & $\mathrm{N}$ & $\mathrm{N}$ & $\mathrm{N}$ & 13 & 13 & G5 & N5 \\
\hline Saturniidae & Hyalphora cecropia & emperor & $\mathrm{Y}$ & $\mathrm{N}$ & $\mathrm{N}$ & 121 & 3 & - & - \\
\hline Satyridae & Neonympha phocion & - & - & - & - & 92 & 13 & - & - \\
\hline Satyridaea & Minois pegala & blue-eyed grayling & - & - & - & 83 & 13 & G5 & - \\
\hline Sphingidae & Agrius cingulata & $\begin{array}{l}\text { pink-spotted hawk } \\
\text { moth }\end{array}$ & $\mathrm{Y}$ & $\mathrm{Y}$ & $\mathrm{Y}$ & 125 & 13 & - & - \\
\hline Sphingidae & Ceratomia undulosa & waved sphinx & $\mathrm{Y}$ & $\mathrm{Y}$ & $\mathrm{Y}$ & 33 & 13 & G5 & N5 \\
\hline Sphingidae & Erinnyis ello & ello sphinx & $\mathrm{N}$ & $\mathrm{Y}$ & $\mathrm{Y}$ & 65 & 17 & G4G5 & N4 \\
\hline Sphingidae & Hemaris diffinis & snowberry clearwing & $\mathrm{Y}$ & $\mathrm{Y}$ & $\mathrm{Y}$ & 9 & 17 & G4G5 & N5 \\
\hline Sphingidae & Hyles gallii & dark-veined deilephila & - & - & - & 118 & 13 & G5 & - \\
\hline Sphingidae & Hyles lineata & white-lined sphinx & $\mathrm{Y}$ & $\mathrm{Y}$ & $\mathrm{Y}$ & 118 & 13 & G5 & N5 \\
\hline Sphingidae & Manduca quinquemaculata & five-spotted sphinx & $\mathrm{Y}$ & $\mathrm{Y}$ & $\mathrm{Y}$ & 125 & 13 & G5 & - \\
\hline Sphingidae & Manduca rustica & six-spotted sphinx & $\mathrm{N}$ & $\mathrm{Y}$ & $\mathrm{Y}$ & 25 & 13 & G5 & - \\
\hline Sphingidae & Manduca sexta & Carolina sphinx & $\mathrm{Y}$ & $\mathrm{Y}$ & $\mathrm{Y}$ & 125,128 & 13 & G5 & - \\
\hline Sphingidae & Paonias excaecata & blind-eyed sphinx & $\mathrm{Y}$ & $\mathrm{Y}$ & $\mathrm{Y}$ & 121 & 13 & G5 & N5 \\
\hline Sphingidae & Paratrea plebeja & plebian sphinx & $\mathrm{Y}$ & $\mathrm{Y}$ & $\mathrm{Y}$ & 33 & 17 & G5 & N5 \\
\hline Sphingidae & Proserpinus juanita & $\begin{array}{l}\text { green-banded day } \\
\text { sphinx }\end{array}$ & $\mathrm{Y}$ & $\mathrm{Y}$ & $\mathrm{Y}$ & 78 & 10 & G4G5 & N4N5 \\
\hline Thyatiridae & Habrosyne scripta & lettered habrosyne & $\mathrm{Y}$ & $\mathrm{Y}$ & $\mathrm{Y}$ & 121 & 3 & G5 & - \\
\hline Thyatiridae & Haematopis grataria & chickweed geometer & $\mathrm{Y}$ & Y & $\mathrm{Y}$ & 48 & 10 & - & - \\
\hline Zanolidae & Apatelodes torrefacta & spotted apatelodes & $\mathrm{Y}$ & $\mathrm{Y}$ & $\mathrm{Y}$ & 121 & 13 & G5 & - \\
\hline
\end{tabular}

${ }^{1}$ Host plant codes from Appendix 1.

${ }^{2}$ Plant utilization codes are from the following references: 1 = Ebner, 1970; 2 = Engelhardt, 1946; 3 = Forbes, 1969; 4 = Hardwick, 1996; $5=$ Howe, 1975; 6 = Klots, 1979; 7 = Opler, 1999; $8=$ Opler and Krizek, 1984; $9=$ Pyle, 1992; $10=$ Richard and Heitzman, 1987; $11=$ Scott, 1986; 12 = Shull, 1987; 13 = Tietz, 1972; 14 = Zhang, 1994; 15 = ipmwww.ncsu.edu/AG271/corn_sorghum/granulate_cutworm.htm; $16=$ www.ext.nodak.edu/extpubs/plantsci/rowcrops/eb25w-6a.htm; 17 = http://www.npwrc.usgs.gov/resource/distr/lepid/BFLYUSA/bflyusa.htm. 


\section{REFERENCES}

Biddinger DJ, Felland CM, Hull LA (1994) Parasitism of tufted apple bud moth (Lepidoptera: Tortricidae) in conventional insecticide and pheromone-treated Pennsylvania apple orchards. Environ. Entomol. 23: 1568-1579

Buchmann SL, Nabhan GP (1996) The Forgotten Pollinators. Island Press, Washington DC

Cardina J, Regnier E, Harrison K (1991) Long-term tillage effects on seed banks in three Ohio USA soils. Weed Sci. 39: 186-194

Engelhardt GP (1946) The North American Clear-wing Moths of the Family Aegeriidae. Bulletin 190. Smithsonian Institution United States National Museum. Washington DC

Ebner JA (1970) The butterflies of Wisconsin. Board of Trustees, Milwaukee Public Museum, Milwaukee, WI

Economic Research Service - USDA (2002) http:// www.ers.usda.gov/Briefing/Corn/background.htm

Foreign Agriculture Service - USDA (2002) http:// www.fas.usda.gov/grain/circular/2002/03-02/cgra_tbl.pdf

Forbes WTM (1969) Lepidoptera of New York and neighboring states. Entomological Reprint Specialists. Lansing, MI

Glenn S, Phillips WH, Kalnay P (1997) Long-term control of perennial broadleaf weeds and triazine-resistant common lambsquarters (Chenopodium album) in no-till corn (Zea mays). Weed Technol. 11: 436-440

Hardwick DF (1996) A monograph to the North American Heliothentinae (Lepidoptera: Noctuidae). Ottawa

Hoffmann MP, Walker DL, Shelton AM (1995) Biology of Trichogramma ostriniae (Hymenoptera: Trichogrammatidae) reared on Ostriniae nubilalis (Lepidoptera: Pyralidae) and survey for additional hosts. Entomophaga 40: 387-402

Howe WH (1975) The Butterflies of North America. Garden City, New York

Johnson KS, Scriber JM, Nitao JK, Smitley DR (1995) Toxicity of Bacillus thuringiensis var. kurtstaki to three nontarget Lepidoptera in field studies. Environ. Ent. 24: 288-297

Julien MH, Griffiths MW (1998) Biological control of weeds: a world catalogue of agents and their target weeds/compiled and edited by Oxon. 4th edn. CABI Publishers, New York

Klots AB (1979) A Field Guide to the Butterflies: North America, east of the Great Plains. Houghton Mifflin. Boston, MA

National Agricultural Statistics Service - USDA (2000) Crop Production: 1999 Summary

NatureServe Explorer: An online encyclopedia of life [web application] (2001) Version 1.6. Arlington, Virginia, USA: NatureServe. Available: http://www.natureserve.org/explorer. (Accessed: October 3, 2002)

Opler PA (1999) Field guide to western butterflies. Houghton Mifflin, Boston, MI

Opler PA, Krizek GO (1984) Butterflies East of the Great Plains: an illustrated natural history. Johns Hopkins University Press. Baltimore, MD
Orr DB, Garcia-Salazar C, Landis DA (2000) Trichogramma nontarget impacts: A method for biological control risk assessment. In Follet, Duan, eds, Nontarget effects of biological control. Kluwer Acad. Publ. pp 111-126

Padgitt M, Newton D, Penn R, Sandretto C (2001) Production Practices for Major Crops in USA Agriculture, 1990-1997. Economic Research Service - USDA

Pavuk DM, Stinner BR (1991) New lepidoptera-parasitoid associations in weedy corn plantings: A potential alternate host for Ostrinia nubilalis (Lepidoptera: Pyralidae) parasitoids. The Great Lakes Entomologist 24: 219-223

Peacock JW, Schweitzer DF, Carter JL, Dubois NR (1998) Laboratory assessment of the effects of Bacillus thuringiensis on native lepidoptera. Environ. Entomol. 27: 450-457

Pyle RM (1992) The Audubon Society field guide to North American butterflies. New York

Richard J, Hietzman JE (1987) Butterflies and moths of Missouri. Missouri Dept. of Conservation, Jefferson City, MO

SAS Institute (1996) SAS/STAT user's guide, release 6.12 ed. SAS Institute, Cary, NC

Schweizer EE, Westra P, Lydecker DW (1998) Seedbank and emerged annual weed populations in cornfields (Zea mays) in Colorado. Weed Technol. 12: 243-248

Scott JA (1986) The butterflies of North America: A natural history and field guide. Stanford University, Stanford, CA

Shull EM (1987) Butterflies of Indiana. Indiana Academy of Science, Bloomington, IN

Tietz HM (1972) North American Lepidoptera. (Vols. 1 and 2). Allyn Museum. Sarasota, FL

U.S. Grains Council (2002) http://www.grains.org/grains/ corn.html

Wax LM, Fwacett RS, Isely D (1981) Weeds of the North Central States, University of Illinois at Urbana-Champaign, Urbana, IL

Webster TM, Cobble HD (1997) Changes in the weed species composition of the southern United States: 1974 to 1995. Weed Technol. 11: 308-318

Western D (1989) Conservation without parks: Wildlife in the rural landscape. In Western D, Pearl M, eds, Conservation for the twenty-first century. Oxford University Press, New York, pp 158-165

Wright MG, Hoffmann MP, Kuhar TP, Pitcher SA, Gardner J, Assessing risks of biological control introductions: a probabilistic risk-assessment approach. (unpublished)

Zhang B (1994) Index of economically important Lepidoptera. CAB International, Wallingford

http://ipmwww.ncsu.edu/AG271/corn_sorghum/ granulate_cutworm.html

http://www.ext.nodak.edu/extpubs/plantsci/rowcrops/eb25w6a.htm

http://www.npwrc.usgs.gov/resource/distr/lepid/BFLYUSA/ bflyusa.htm 